3 Dorsch JA, Dorsch SE. Equipment checking and maintenance. In: Dorsch JA, Dorsch SE (Eds). Understanding Anesthesia Equipment, $4^{\text {th }} \mathrm{ed}$. Baltimore: Williams and Wilkins;1999: 938.

4 Cooper JB, Newbower RS, Kitz RJ. An analysis of major errors and equipment failures in anesthesia management: consideration for prevention and detection. Anesthesiology 1984; 60: 34-42.

5 Kumar V, Barcellos WA, Mehta MP, Carter JG. An analysis of critical incidents in a teaching department for quality assurance. A survey of mishaps during anaesthesia. Anaesthesia 1988; 43: 879-83.

\section{Insertion of a nasogastric tube with the patient in the prone position}

To the Editor:

Although many techniques to facilitate the passage of a nasogastric tube (NGT) have been reported, the insertion of an NGT in anesthetized patients can at times be unexpectedly difficult. ${ }^{1-3}$ We evaluated a novel approach of using the prone position to facilitate NGT insertion compared with classic insertion in the supine position. The Hospital Ethics Committee approved this protocol, and informed consent was obtained from 45 patients scheduled for elective lumbar supine surgery. After orotracheal intubation under general anesthesia, a 14-French silicon NGT (Create Medic Company, Ltd., Yokohama, Japan) was generously lubricated and inserted in the supine position. The anesthesiologist was permitted to lift the patient's tongue jaw, the cricoid cartilage and the thyroid cartilage. The right nostril was filled with the lidocaine jelly, and the NGT was gently inserted into the naris and blindly advanced into the stomach. If the NGT coiled or failed to advance into the stomach on the first attempt, the NGT was withdrawn slightly and secondary insertion was attempted. Placement of the NGT in the stomach was confirmed by the presence of a swooshing sound heard through a stethoscope placed over the stomach while injecting $10 \mathrm{~mL}$ of air into the NGT. After a successful insertion or failure twice in the supine position, the NGT was withdrawn slightly, and the patient was turned to the prone Hall-frame position on a lumbar supine surgery table. The patient's neck was rotated $45^{\circ}$ to the right on the table and the NGT was inserted. The number of successful inserted cases within two attempts at either position was compared. In one patient, neck lateral $x$ ray imaging was taken in both positions.

The number of successful cases in the prone position $(42 / 45,93 \%)$ was significantly higher than in the

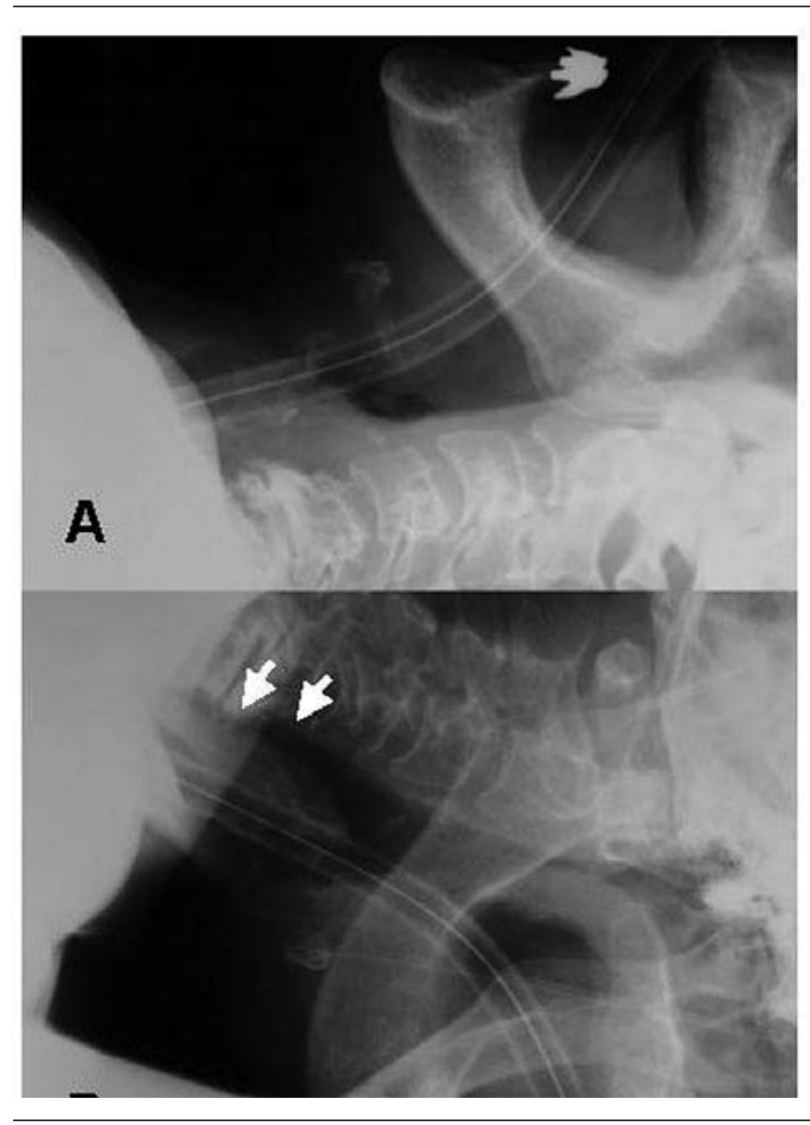

FIGURE Cervical $x$-ray imaging of a case in which a nasogastric tube (NGT) could not be advanced into the stomach with the patient in the supine position (A), but could be advanced with the patient in the prone position (B). There was obviously a wide space (arrow) to pass an NGT at the hypopharynx and esophageal constrictions in the prone position, but no space in the supine position.

supine group $(15 / 45,33 \%),(P<0.01)$. Cervical $x$-ray imaging (Figure) shows that there was sufficient space to pass an NGT at the hypopharynx and esophageal constrictions in the prone position. In this patient, the NGT was coiled at the pharynx, and did not advance to the esophagus in the supine position. However, in the prone position, the NGT was easily advanced into the stomach.

Ozer et al. ${ }^{1}$ reported that the arytenoid cartilages and piriform sinuses resist passage of an NGT at the laryngeal level. Isono et al. ${ }^{4}$ reported in patients with obstructive sleep apnea that the supine position not only decreased static pressure-area curves and maximum cross-sectional area, but also increased the closing pressure at both the retropalatal and retroglossal airways. We report that the prone position facilitates the insertion of an NGT compared with the supine 
position, and suggest that the prone position would give wider space to the hypopharynx and upper esophagus than the supine position under general anesthesia with muscle relaxant. We recognize the indications for this prone position method are limited. However, an NGT easily advances into the stomach almost uniformly on the first attempt in the prone position, and this simple and fast technique may be useful in certain clinical situations.

Masanori Yamauchi MD PhD

Shingo Furuse MD

Makoto Asano MD PhD

Masanori Watanabe MD

Soushi Iwasaki MD

Akiyoshi Namiki MD PhD

Sapporo Medical University School of Medicine, Hokkaido, Japan

E-mail: my3@rb4.so-net.ne.jp

\section{References}

1 Ozer S, Benumof JL. Oro- and nasogastric tube passage in intubated patients: fiberoptic description of where they go at the laryngeal level and how to make them enter the esophagus. Anesthesiology 1999; 91: 137-43.

2 Shetty S, Henthorn RW, Ganta R. A method to reduce nasopharyngeal trauma from nasogastric tube placement (Letter). Anesth Analg 1994; 78: 410-1.

3 Mundy DA. Another technique for insertion of nasogastric tubes (Letter). Anesthesiology 1979; 50: 374.

4 Isono S, Tanaka A, Nishino T. Lateral position decreases collapsibility of the passive pharynx in patients with obstructive sleep apnea. Anesthesiology 2002; 97 : 780-5.

\section{When general anesthesia is required to remove an epidural catheter}

To the Editor:

We present a case wherein an entrapped epidural catheter required general anesthesia with muscle relaxation for removal.

A 40-yr-old, 160-cm, and 50-kg woman was scheduled for transabdominal hysterectomy and oophorectomy. With the patient in the right lateral position, a 19-G Arrow Flextip Plus ${ }^{\mathrm{TM}}$ (Arrow International, Reading, PA, USA) epidural catheter was inserted at the T11-12 interspace via a median approach. The catheter was secured to the skin at the $10-\mathrm{cm}$ mark. The epidural catheter was used successfully for anesthesia during surgery and for analgesia after sur- gery. On the second day, resistance was encountered upon trying to remove the catheter. An abdominal roentgenogram showed neither knotted nor looped catheter. The catheter could be withdrawn to the 7-cm mark at the skin by repeated removal attempts in varied positions. However, a stretched and fragile part was found in the catheter. We explained the risk of further attempts with limited flexed positioning, and obtained informed consent for general anesthesia. Thiamylal and vecronium were administered to facilitate oral intubation, and general anesthesia was maintained with sevoflurane and nitrous oxide. The patient was placed in the right lateral position with her back maximally flexed, and the catheter could then be removed without breaking.

A fundamental rule for removing an entrapped epidural catheter is to place the patient in the same position as they were at the time of its insertion. However, the lateral position is better than sitting, because more than twice as much force is required to remove the catheter when the patient is sitting. ${ }^{1}$ Radiography is important for the early diagnosis. An electronic scale might be also useful. The force required to remove an epidural catheter does not exceed $0.6 \mathrm{~kg}$ in usual cases. ${ }^{2}$ The fracture force of Arrow and Portex catheters are about 2 and $2.6 \mathrm{~kg}$, respectively. ${ }^{2,3}$ Other strategies to facilitate removal include: 1 ) discontinue efforts temporarily to allow tissue relaxation; 2) tie a weight to the catheter and let it hang down; 3 ) withdraw the catheter under sedation; 4) thread a sterile Tuohy needle over the catheter into the epidural space and withdraw them en bloc and; 5) inject saline through the catheter to widen and lubricate the surrounding space. ${ }^{4}$ Never risk damaging the entrapped catheter. Special attention was given to the Arrow catheter in our patient because it has a weak segment between the 7 - and 8 -cm marks. ${ }^{5}$

In conclusion, we found that general anesthesia was an effective option to remove an entrapped epidural catheter, because it completely alleviated the pain of the surgical wound, relaxed the muscles, and enabled the patient to flex maximally.

Taeko Fukuda MD*

Satoru Iijima MD $\dagger$

Noriko Takayama MD†

Tsuyoshi Satsumae MD‡

Shigeyuki Saito MD

Hidenori Toyooka MD*

Tsukuba University, ${ }^{*}$ Ushiku Aiwa General

Hospital, $†$ Tsukuba-Gakuen Hospital, $\ddagger$

Tsukuba, Ibaraki, Japan

E-mail: taekof@md.tsukuba.ac.jp 